\title{
Humoral and cellular immune responses to influenza vaccination in children with cancer receiving chemotherapy
}

\author{
ROSA MARÍA WONG-CHEW ${ }^{1}$, MARGARITA NAVA FRÍAS ${ }^{2}$, MIGUEL LEONARDO GARCÍA-LEÓN ${ }^{1}$, \\ LOURDES ARRIAGA-PIZANO ${ }^{3}$, AURORA MEDINA SANSON ${ }^{2}$, CONSTANTINO LOPEZ-MACÍAS $^{3}$, \\ ARMANDO ISIBASI $^{3}$ and JOSÉ IGNACIO SANTOS-PRECIADO ${ }^{1}$ \\ ${ }^{1}$ School of Medicine, Department of Experimental Medicine, National Autonomous University of Mexico; \\ ${ }^{2}$ Oncology and Infectious Diseases Departments, Children's Hospital of Mexico 'Federico Gómez'; \\ ${ }^{3}$ Medical Research Unit on Immunochemistry (UIMIQ), Specialties Hospital, National Medical Centre \\ 'Siglo XXI', Mexican Social Security Institute (IMSS), Mexico City, Mexico
}

Received January 30, 2012; Accepted April 13, 2012

DOI: $10.3892 / \mathrm{ol} .2012 .721$

\begin{abstract}
The immune response to influenza vaccination in children with cancer is controversial. The objective of this study was to characterize the cellular and humoral immune responses to an influenza vaccine in children with cancer who were receiving chemotherapy. In this study, children with cancer, who were not previously immunized, received an influenza vaccine via intramuscular injection. Blood samples were obtained prior to and at 4 weeks after immunization. Antibodies were measured using a hemagglutination inhibition (HI) assay. Cell-mediated immunity was measured by specific lymphoproliferation with ${ }^{3} \mathrm{H}$-thymidine incorporation and by measuring cell frequencies following staining with monoclonal antibodies (CD8, CD4, CD19, CD45RA and CD27) using flow cytometry following incubation with the influenza antigen for 5 days. Geometric mean titers (GMT), mean counts per minute (cpm), cell frequencies prior to and following vaccination and percentage patient responses were compared using the Mann-Whitney non-parametric U and Chi-square tests; where $\mathrm{p}<0.05$ was considered to indicate a statistically significant result. A total of 56 children were included. Their mean age was $6.64 \pm 3.61$ years. Acute lymphoblastic leukemia (ALL) was diagnosed in 75, solid tumors in 23 and lymphoma in $2 \%$ of the children. Subjects with titers $\geq 40$ hemagglutination units (HU) increased from $43 \%$ prior to vaccination to $73 \%$ following vaccination $(\mathrm{p}=0.01)$, whereas the GMT increased from 31.35 [95\% confidence interval (CI), 29-111] to $143.45 \mathrm{HU}$ (95\% CI,
\end{abstract}

Correspondence to: Dr Rosa María Wong-Chew, Departamento de Medicina Experimental, Facultad de Medicina, UNAM; Hospital General de México, Dr. Balmis \#148, Colonia Doctores 06726, Mexico

E-mail: rmwong@unam.mx

Key words: influenza vaccine, children, cancer, chemotherapy
284-640) following vaccination $(\mathrm{p}<0.001)$. An increase in CD45RA expression in $\mathrm{CD}^{+} \mathrm{T}$ cells was observed following vaccination $(\mathrm{p}=0.01)$. An increase in $\mathrm{CD} 27$ expression was observed in the CD4/8-negative cell population stimulated with the influenza antigen following vaccination $(\mathrm{p}<0.05)$. No serious adverse effects were observed. An increase in the seropositivity rate and GMT values following influenza vaccination were also observed. Influenza immunization was well tolerated among these children with cancer and increased the humoral and cellular immune responses with the activation of probable lymphoid precursors.

\section{Introduction}

The influenza virus is a pathogen that causes respiratory disease in humans and has the potential to cause epidemics and pandemics (1). During the 1918 pandemic, 40-50 million individuals succumbed to the disease globally (2). During the 2009 pandemic, 17,483 mortalities were reported to the World Health Organization with an estimate of 200 million H1N1 influenza cases worldwide for December 2010 (3). In the United States, influenza is more frequent in winter and is associated with 36,000 mortalities annually (4). In Mexico, influenza has been associated with $7-12 \%$ of respiratory infections in certain areas $(5,6)$.

Influenza infection is characterized by sudden respiratory symptoms (fever, myalgia, headache, coughing, pharyngeal aching and rhinitis) (7). The uncomplicated disease improves within 3-7 days. However, in certain individuals with risk factors, complications may present including viral pneumonia, secondary bacterial infections or coinfections, sepsis and even mortality. In young children, influenza infection may present as sepsis with a high fever (8). The risk factors include pneumopathy, cardiopathy and an immunocompromised state that may be observed among patients with cancer or human immunodeficiency virus (HIV) and steroid recipients (4,9-16).

Chemotherapy inhibits the ability of the immune system to develop an immune response to invading pathogens via vaccination or incidental exposure and acts by suppressing bone 
marrow production and decreasing the vital components of the immune system (17). There is controversy with regard to the humoral immune response to influenza vaccination in children with cancer who are receiving chemotherapy. Certain authors have reported an effective antibody response in such children, albeit at a lower proportion than among healthy individuals, especially when receiving chemotherapy at the moment of vaccination $(18,19)$.

Certain studies have suggested that cellular immunity to an influenza vaccine is a useful predictor of protection against disease in the elderly (20). The cellular immune response to an influenza vaccine has been described as the expansion of $\mathrm{CD}^{+}$and $\mathrm{CD}^{+} \mathrm{T}$ lymphocytes with surface markers that classify them as effector or memory $\mathrm{T}$ cells based on the surface markers CD62L and CD45RA (21-24); other molecules, including CD27, are costimulatory and indicate activation (25). These surface markers have yet to be characterized in children with cancer who are receiving chemotherapy and who have been immunized with an influenza vaccine. The objective of this study was to characterize the cellular and humoral immune responses to influenza vaccination among children with cancer who are receiving chemotherapy.

\section{Materials and methods}

Ethics. This study was a prospective, quasi-experimental, comparative clinical trial with auto controls. The study was approved by the Institutional Review Board of the Hospital Infantil de México Federico Gómez (Mexico City, Mexico; HIM/2007/025) and the School of Medicine of the Universidad Nacional Autónoma de México (Mexico City, Mexico; 19-2007). Informed consent forms were signed by the parents or guardians of all the study participants. The study was conducted following the Good Clinical Practice and the International Conference of Harmonization standards.

Study population. The patients included in the study were 56 individuals with cancer who were aged 2-16 years and were receiving chemotherapy at the Oncology Department of the Hospital Infantil de México Federico Gómez, Mexico. Exclusion criteria were an allergy to egg protein, thrombocytopenia or the lack of a follow-up blood collection. The subjects received the inactivated trivalent influenza vaccine prior to chemotherapy administration. No participant had a history of influenza vaccination. Blood samples were obtained prior to and at 4 weeks after vaccination for humoral and cellular assays. The clinical symptoms were assessed over 14 days and on day 30 .

Influenza vaccine and vaccine administration. Inactivated trivalent Vaxigrip vaccine (Sanofi Pasteur, Paris, France), containing $15 \mu \mathrm{g}$ of hemagglutinin protein for each virus [A/ New Caledonia/20/99 (H1N1), A/Wisconsin/67/2005 (H3N2) and B/Malaysia/2560/2004] was used for the winter season of 2006-2007. Fluarix vaccine (GlaxoSmithKline, Mexico) containing $15 \mu \mathrm{g}$ of hemagglutinin protein for each virus [A/Solomon Islands/3/2006 (H1N1), A/Wisconsin/67/2005 (H3N2) and B/Malaysia/2560/2004] was used for the winter season of 2007-2008. The vaccine was administered intramuscularly over the deltoid area of the left arm.
Influenza antibody assay. IgG anti-influenza antibodies were detected by the hemagglutination inhibition (HI) technique. Briefly, the serum was separated by centrifugation and frozen at $-70^{\circ} \mathrm{C}$ until tested in parallel. Aliquots of serum $(50 \mu \mathrm{l})$ treated with enzyme-destroying receptor overnight in several dilutions were placed in duplicate 24 -well plates. A concentration of 8 hemagglutination units (HU) for each virus (A H1N1, A H3N2 and B) was added for 30 min. A $0.85 \%$ suspension of turkey erythrocytes was added for $20 \mathrm{~min}$ until inhibition of hemagglutination was observed. A titer of $\geq 1: 40$ was considered a positive response.

Cell proliferation measured by ${ }^{3} H$-thymidine. Fresh peripheral blood mononuclear cells (PBMC) were separated from whole blood by Ficoll-Hypaque gradients and added to 96-well microtiter plates at a concentration of $3 \times 10^{5}$ cells/ well in RPMI-1640 medium (Gibco, Gaithersburg, MD, USA) containing 10\% normal human serum (Sigma-Aldrich, St. Louis, MO,USA). The influenza antigen, prepared from infected Madin-Darby Canine Kidney (MDCK) cell lysates or an uninfected cell control, was added at dilutions of 1:4, 1:8 and 1:16 in triplicate wells. T-cell proliferation was measured by adding ${ }^{3} \mathrm{H}$-thymidine $(2.5 \mu \mathrm{Ci}$ per well) after 5 days for $6-18 \mathrm{~h}$. The stimulation index (SI) was calculated as the mean counts per minute (cpm) in the influenza antigen-stimulated wells divided by the mean cpm in the control wells. Phytohemagglutinin (Difco, Detroit, MI, USA) and tetanus toxoid (Calbiochem, La Jolla, CA, USA) were used as positive controls.

Cell populations measured by flow cytometry. Separated PBMC were added to 96 -well microtiter plates at a concentration of $3 \times 10^{5}$ cells/well in RPMI-1640 (Gibco) with $10 \%$ normal human serum (Sigma-Aldrich). The cells were stimulated with the inactivated influenza antigen or an uninfected cell control (MDCK cells); concanavalin A was used as a mitogen control. After 5 days, the cells were stained with monoclonal antibodies conjugated to CD8-Pe-Cy7, CD4-PeCy5 and CD19-PE cells and with markers related to activation and memory CD45RA-PE and CD27-FITC cells (BD Pharmigen, La Jolla, CA, USA). The cell frequencies were measured using flow cytometry in the FacsCanto II cytometer (Becton Dickinson and Company, Franklin Lakes, NJ, USA).

Statistical analysis. Geometric mean titers (GMT), mean cpm values and the mean cell frequencies prior to and at 1 month after vaccination were compared using the non-parametric Mann-Whitney U test. The percentages were compared using Chi-square tests and $\mathrm{p}<0.05$ was considered to indicate a statistically significant result. Results are expressed as the means \pm standard deviation (SD) and the antibody response as geometric mean titers with $95 \%$ confidence intervals (CI).

\section{Results}

Study population and clinical characteristics. A total of 56 children were included in the study, recruited in the winter seasons between December 2006 and February 2007 (33 individuals) and between October 2007 and February 2008 (23 individuals). The mean age $( \pm \mathrm{SD})$ was $6.6 \pm 3.6$ years, the mean weight was $22.4 \pm 10.1 \mathrm{~kg}$ and the mean height was 
A

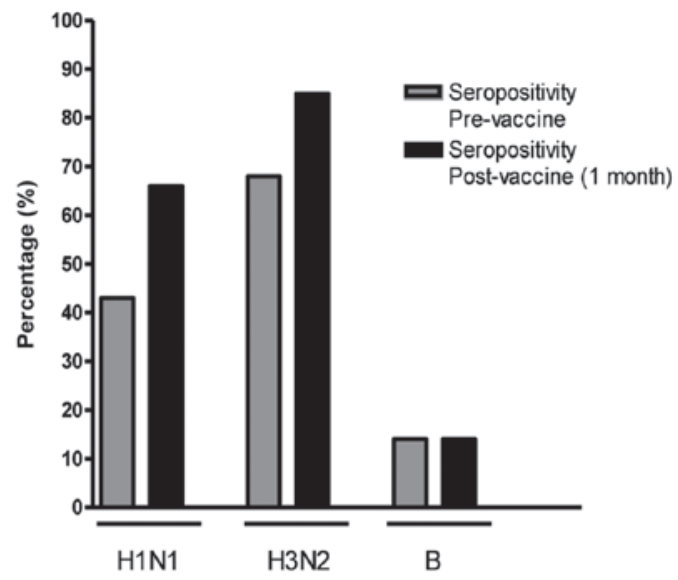

B

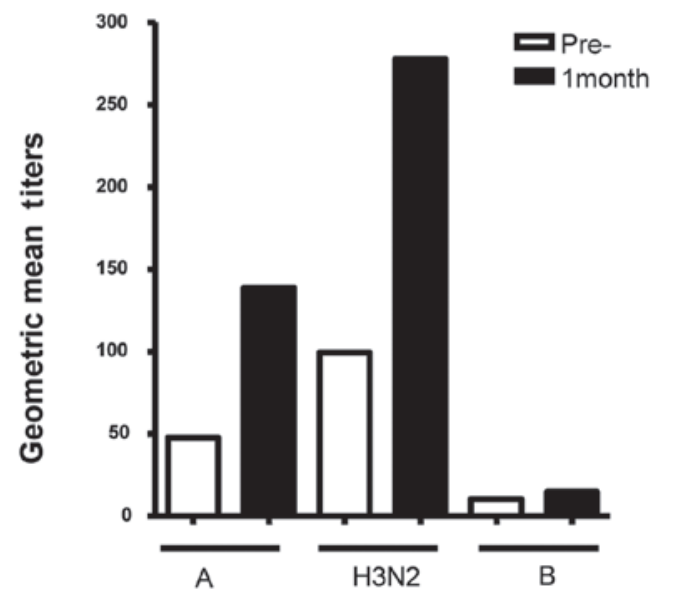

Figure 1. Humoral immunity. (A) Increases in the percentage of seropositive patients and (B) geometric mean viral titers observed for serotypes A H1N1, A H3N2 and B at 1 month after the influenza vaccination.

$1.1 \pm 0.2 \mathrm{~m}$. The underlying diagnosis was acute lymphoblastic leukemia (ALL) in 75, solid tumors in 23 and lymphoma in $2 \%$ of the patients. All 56 children were receiving chemotherapy at the moment of vaccination. Among the patients with ALL, 19\% were on the induction phase and $81 \%$ on the maintenance phase of chemotherapy. Of the 56 patients, $25 \%$ were on one chemotherapy drug, 40 on two, 16 on three, 16 on four and 3 on five. The majority of patients were receiving either methotrexate or vincristine alone or in combination with other chemotherapy agents: 53\% received vincristine, $47 \%$ methotrexate, $19 \%$ cytarabine, $19 \%$ daunorubicin, 25\% L-asparaginase, $15 \%$ cyclophosphamide, $15 \%$ mercaptopurine, $15 \%$ dexamethasone, $6 \%$ etoposide, $6 \%$ actinomycin and $3 \%$ carboplatin.

Antibody response to influenza vaccine. Seropositivity for the three serotypes (H1N1, H3N2 and B) prior to vaccination was observed in $43 \%$ of the patients and an increase to $73 \%$ was observed 1 month after vaccination. An individual analysis for each serotype of the virus at 1 month after vaccination demonstrated an increase in seropositivity from $43 \%$ prior to vaccination to $63 \%$ for the H1N1 serotype ( $\mathrm{p}=0.02)$, from 68 to $85 \%$ following vaccination for the H3N2 serotype ( $\mathrm{p}=0.05)$ and from 0 to $14 \%$ for the B serotype $(\mathrm{p}=0.006$; Fig. 1$)$. An increase in the GMT was observed from 31.35 (95\% CI, 29-111) prior to vaccination to 143.45 (95\% CI, 284-640) 1 month after immunization for all three serotypes $(\mathrm{p}<0.001)$. An individual analysis by serotype revealed an increase in the GMT for the H1N1 influenza virus from 47 (95\% CI, 128-378) prior to vaccination to 138 (95\% CI, 363-685) following immunization $(\mathrm{p}=0.009)$ and for the $\mathrm{H} 3 \mathrm{~N} 2$ virus from 99 (95\% CI, 208-485) to 277 (95\%, CI, 466-775; $\mathrm{p}=0.009)$. This contrasted with the influenza B virus, where no statistically significant increase in the GMT was observed, ranging from 10 (95\% CI, 9-10) prior to vaccination to 14 (95\% CI, 5-58) following immunization $(\mathrm{p}=0.11)$.

Cell-mediated immune response to influenza vaccine. We did not observe any increase in the mean SI value of $1.19 \pm 0.70$ prior to vaccination compared with $1.65 \pm 1.03$ following influenza immunization $(\mathrm{p}=0.31)$ using the lymphoproliferation assay measured by ${ }^{3} \mathrm{H}$-thymidine (Fig. 2A). No differences were observed in the percentages of $\mathrm{CD}^{+}$and $\mathrm{CD} 8^{+} \mathrm{T}$ cells prior to and following vaccination with or without influenza antigen stimulation. However, an increase was observed in the expression of CD45RA in $\mathrm{CD} 4^{+} \mathrm{T}$ cells with influenza antigen stimulation $(0.07 \pm 0.05$ without stimulation vs. $2.83 \pm 1.46$ stimulated; $\mathrm{p}<0.001)$ and in $\mathrm{CD} 8^{+} \mathrm{T}$ cells $(0.29 \pm 0.24$ without stimulation vs. $6.80 \pm 1.74$ stimulated; $p<0.001)$ prior to vaccination and in $\mathrm{CD} 8^{+} \mathrm{T}$ cells following vaccination $(0.18 \pm 0.15$ without stimulation vs. $3.37 \pm 1.65$ stimulated; $p=0.01$; Fig. $2 \mathrm{~B}$ ). An increase in expression of the CD27 molecule was observed in $\mathrm{CD} 4 \% \mathrm{CD} 8$ - cells when stimulated with influenza antigen prior to vaccination $(0.01 \pm 0.007$ without stimulation vs. $3.15 \pm 0.96$ stimulated; $p<0.0001)$, as well as following vaccination $(0.21 \pm 0.16$ without stimulation vs. $10.44 \pm 3.58$ stimulated; $\mathrm{p}<0.0001)$. A difference in the expression of CD27 with influenza stimulation prior to vaccination was observed $(3.15 \pm 0.96$ vs. $10.44 \pm 3.58$ following vaccination; $p=0.05$; Fig. 2 C). These CD4/CD8 cells were also CD56/CD16 (data not shown).

Adverse effects. The vaccine was well tolerated by the individuals; no serious adverse effects were reported and no hospitalizations due to the influenza vaccination were reported. Most individuals presented with local symptoms: $70 \%$ with pain, $9 \%$ with erythema, $4 \%$ with swelling and $2 \%$ with redness in the injection area. General symptoms included $5 \%$ of children presenting with fever, $2 \%$ with rhinorrhea, $2 \%$ with malaise, $4 \%$ with myalgias, $5 \%$ with headache and $2 \%$ with coughing. The general symptoms resolved spontaneously within days following the administration of symptomatic treatment.

\section{Discussion}

Influenza is a significant disease worldwide due to its pathogenic characteristics and its ability to constantly change external surface proteins. The virus can cause severe disease and has the potential to cause epidemics and pandemics even when patients have had previous contact with related strains of the virus $(26,27)$. A notable example is the 2009 influenza type A H1N1 pandemic first reported in Mexico. This pandemic was caused by a novel recombinant virus, with atypical manifestations in the first reported cases that developed fatal pneumonia (28-32). The disease is usually mild in healthy individuals, but immunocompromised patients may develop severe pneumonia and secondary bacterial infections and can 
A

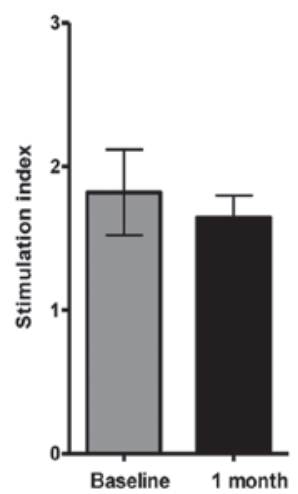

B

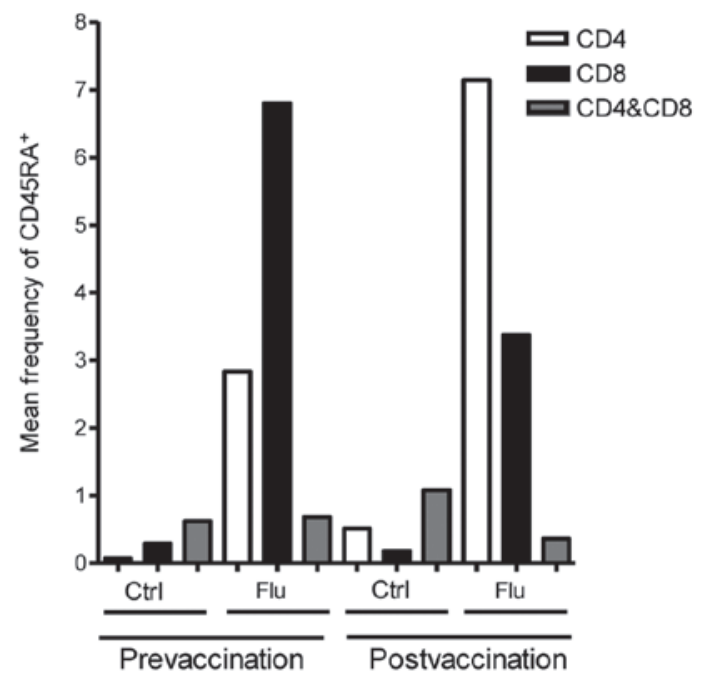

C

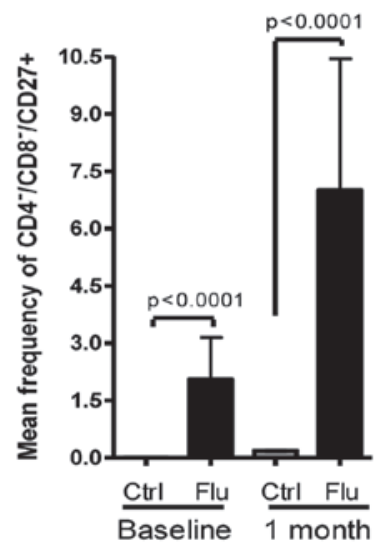

Figure 2. Cell-mediated immunity. (A) No differences were observed after vaccination measured by the ${ }^{3} \mathrm{H}$-thymidine assay for cell proliferation. (B) An increase in the percentage of CD45RA ${ }^{+}$cells was observed 1 month after vaccination in the $\mathrm{CD} 8^{+}$and $\mathrm{CD} 4^{+}$cell lineages stimulated with influenza antigen. (C) An increase in the mean frequency of CD4 $/ \mathrm{CD} 8 / \mathrm{CD} 27^{+}$cells (probable precursors of the lymphoid lineage) was observed with influenza antigen stimulation, prior to and following vaccination. The figure shows flow cytometry plots for one representative patient.

succumb to the disease. Due to the potential severe disease and the associated complications, influenza vaccination is recommended annually for this high-risk population $(26,27)$.

In Mexico, there have been few studies concerning influenza infections prior to the 2009 pandemic and the data are scarce. The influenza surveillance network (FluNet) from the World Health Organization reported 97 cases of influenza virus in Mexico in 2006, 384 in 2007 and 118 in 2008; in contrast to 81,701 cases detected during the 2009 pandemic and 2,878 until June 2010 (33). Two groups in Mexico reported incidence of influenza prior to the pandemic of $7-12 \%(5,6)$. Although the present study had a small sample size, it was observed that $43 \%$ of the subjects expressed antibodies against influenza types A H1N1, A H3N2 and B prior to vaccination; this proportion increased to $73 \%$ following vaccination. The observation of $43 \%$ seropositivity prior to vaccination suggests an underreporting of influenza cases, the circulation of the virus and previous infection since no study participants had a history of influenza vaccination. Although these children were receiving chemotherapy during immunization, the antibody response increased following influenza vaccination to $73 \%$. This is in contrast to the results of a previous study which suggested that chemotherapy limits the ability to develop a sufficient immune response to vaccination and reported a seropositivity level of 32\% following vaccination (19).

An increase in the level of antibodies was observed 1 month after vaccination, in the absence of a cellular immune response detected by the ${ }^{3} \mathrm{H}$-thymidine cell proliferation assay. However, an increase in the expression of CD45RA ${ }^{+}$in $\mathrm{CD}^{+}$and $\mathrm{CD} 8^{+}$ cells prior to vaccination and $\mathrm{CD} 8^{+}$cells following vaccination was detected, suggesting activation prior to vaccination. CD45 in combination with CCR7 in CD8 ${ }^{+}$cells are markers of naïve cells $\left(\mathrm{CD}^{2} 5 \mathrm{RA}^{+} \mathrm{CCR}^{+}\right)$, effector cells $\left(\mathrm{CD} 45 \mathrm{RA} \mathrm{A}^{-} /\right.$ CCR7 $7^{-}$, central memory cells (CD45RA $/ \mathrm{CCR}^{+}$), effector memory cells (CD45RA/CCR7') or RA effector memory cells (CD45RA $\left.{ }^{+} / \mathrm{CCR}^{-}\right)$(24). CD45RA alone with CD4 and CD8 are naïve cells, which react with a new antigen. This explains the increase in these cells prior to vaccination when stimulated with influenza virus. $\mathrm{CD}^{+} / \mathrm{CD} 45 \mathrm{RA}^{+} \mathrm{T}$ cells increased with influenza stimulation following vaccination, contrary to a decrease observed in $\mathrm{CD}^{+} / \mathrm{CD} 45 \mathrm{RA}^{+} \mathrm{T}$ cells stimulated after vaccination. The decrease in the two naïve populations may be explained by the effect of the chemotherapy agents, but the increase in the $\mathrm{CD} 4$ population may suggest a faster recovery of the naïve $\mathrm{CD} 4 \mathrm{~T}$ cells rather than naïve $\mathrm{CD} 8 \mathrm{~T}$ cells.

An increase in the expression of CD27 was also observed in $\mathrm{CD} 4 / \mathrm{CD} 8 / \mathrm{CD} 19^{-}$cells when stimulated with influenza antigen and expression was higher following vaccination. These cells were CD56 to rule out natural killer cells (data not shown) and were then gated in the lymphocyte area in a flow cytometry forward side scatter plot, suggesting the activation of a precursor of T or B cells. Mature lymphocytes did not express this marker, only the $\mathrm{CD}^{-} / \mathrm{CD} 8^{-}$cells. Staining the cells with other precursor markers may have yielded noteworthy results, but the present study did not observe any increase in this population until the recruitment of the patients was already finished.

The lack of lymphoproliferative responses of mature lymphocytes, measured by ${ }^{3} \mathrm{H}$-thymidine and by flow cytometry, may be explained by the constant assault on the cells induced by chemotherapy. Chemotherapy is cytotoxic to lymphocytes and to the bone marrow (17). The bone marrow increases the production of blood cells that enter the circulation and precursors are located within the peripheral blood of children undergoing chemotherapy. This may explain the presence of lymphocyte precursors. However, in this study, the lymphocyte precursors associated with the activation marker CD27 (data not reported previously). Although chemotherapy 
is toxic to bone marrow and lymphocytes, the present study revealed that $43 \%$ of patients expressed influenza-specific antibodies prior to vaccination. This suggests that memory $\mathrm{B}$ cells or memory plasma cells in the bone marrow are not fully affected by chemotherapy.

These results suggest that immunization against influenza in children with cancer who are receiving chemotherapy may increase the seropositivity rates, the GMT values and cellular immune responses with the activation of probable lymphoid precursors. The influenza vaccine was well tolerated in this population.

\section{Acknowledgements}

We are grateful for the generous donation of influenza vaccine from GlaxoSmithKline, Mexico. We are grateful to the participants of the study and their parents and guardians, to the social worker Juana Rodríguez-Castillo and to the pediatric fellows at the Department of Infectious Disease Eneida SanchezMedina and Mildred Zambrano-Leal for their assistance with the patients' recruitment. This study was supported in part by a grant from the Fogarty International Center and the Office of Research on Women's Health (TW006193) and in part by a grant from the Federal Funds of the Hospital Infantil de México Federico Gómez (CO/2007/038). This study was presented as an abstract at the 48th Annual Interscience Conference on Antimicrobial Agents and Chemotherapy (ICAAC) and the Infectious Disease Society of America (IDSA) 46th Annual Meeting, Washington, DC, USA, October 2008 and the annual meeting of the Asociación Mexicana de Infectología y Microbiología, León, Guanajuato, México, May 2008.

\section{References}

1. Wright PF and Webster RG: Orthomyxoviruses. In: Fields Virology. Knipe DM and Howley PM (eds). 4th edition.Lippincott Williams \& Wilkins, Philadelphia, pp1534-1579, 2001.

2. Cox NJ and Subbarao K: Influenza. Lancet 354: 1277-1282, 1999.

3. Girard MP, Tam JS, Assossou OM and Kieny MP: The 2009 A (H1N1) influenza virus pandemic: A review. Vaccine 28: 4895-4902, 2010.

4. Thompson WW, Shay DK, Weintraub E, et al: Mortality associated with influenza and respiratory syncytial virus in the United States. JAMA 289: 179-186, 2003.

5. Ayora-Talavera G, Gongora-Biachi RA, Lopez-Martinez I, et al: Detection of human influenza virus in Yucatan, Mexico. Rev Invest Clin 54: 410-414, 2002.

6. Noyola DE, Rodriguez-Moreno G, Sanchez-Alvarado J, Martinez-Wagner R and Ochoa-Zavala JR: Viral etiology of lower respiratory tract infections in hospitalized children in Mexico. Pediatr Infect Dis J 23: 118-123, 2004.

7. Nicholson KG: Clinical features of influenza. Semin Respir Infect 7: 26-37, 1992.

8. Dagan R and Hall CB: Influenza A virus infection imitating bacterial sepsis in early infancy. Pediatr Infect Dis 3: 218-221, 1984.

9. Thompson WW, Shay DK, Weintraub E, et al: Influenzaassociated hospitalizations in the United States. JAMA 292: 1333-1340, 2004.
10. Barker WH and Mullooly JP: Impact of epidemic type A influenza in a defined adult population. Am J Epidemiol 112: 798-811, 1980.

11. Lui KJ and Kendal AP: Impact of influenza epidemics on mortality in the United States from October 1972 to May 1985. Am J Public Health 77: 712-716, 1987.

12. Eickhoff TC, Sherman IL and Serfling RE: Observations on excess mortality associated with epidemic influenza. JAMA 176: 776-782, 1961 .

13. Barker WH and Mullooly JP: Pneumonia and influenza deaths during epidemics: implications for prevention. Arch Intern Med 142: 85-89, 1982

14. Simonsen L, Clarke MJ, Schonberger LB, Arden NH, Cox NJ and Fukuda K: Pandemic versus epidemic influenza mortality: a pattern of changing age distribution. J Infect Dis 178: 53-60, 1998.

15. Keren R, Zaoutis TE, Bridges CB, et al: Neurological and neuromuscular disease as a risk factor for respiratory failure in children hospitalized with influenza infection. JAMA 294: 2188-2194, 2005.

16. Grijalva CG, Craig AS, Dupont WD, et al: Estimating influenza hospitalizations among children. Emerg Infect Dis 12: 103-109, 2006.

17. Balmer CM, Valley AW and Iannucci A: Cancer treatment and chemotherapy. In: Pharmacotherapy: A Pathophysiologic Approach. DiPiro JT, Talbert RL, Yee GC, Matzke GR, Wells BG, Posey M and Schwinghammer T (eds). 6th edition. McGraw-Hill, New York, NY, pp2279-2328, 2005.

18. Anderson H, Petrie K, Berrisford C, Charlett A, Thatcher N and Zambon M: Seroconversion after influenza vaccination in patients with lung cancer. Br J Cancer 80: 219-220, 1999.

19. Matsuzaki A, Suminoe A, Koga Y, Kinukawa N, Kusuhara K and Hara T: Immune response after influenza vaccination in children with cancer. Pediatr Blood Cancer 45: 831-837, 2005.

20. McElhaney JE, Xie D, Hager WD, et al: T cell responses are better correlates of vaccine protection in the elderly. J Immunol 176: 6333-6339, 2006.

21. Woodland DL: Cell-mediated immunity to respiratory virus infections. Curr Opin Immunol 15: 430-435, 2003.

22. Kim SV and Flavell RA: Immunology. CD8alphaalpha and T cell memory. Science 304: 529-530, 2004.

23. Kaech SM, Wherry EJ and Ahmed R: Effector and memory T-cell differentiation: implications for vaccine development. Nat Rev Immunol 2: 251-262, 2002.

24. Robinson HL and Amara RR: T cell vaccines for microbial infections. Nat Med 11: S25-S32, 2005.

25. Matter MS, Claus $\mathrm{C}$ and Ochsenbein AF: $\mathrm{CD}^{+} \mathrm{T}$ cell help improves $\mathrm{CD} 8^{+} \mathrm{T}$ cell memory by retained $\mathrm{CD} 27$ expression. Eur J Immunol 38: 1847-1856, 2008.

26. Smith NM, Bresee JS, Shay DK, Uyeki TM, Cox NJ and Strikas RA: Prevention and Control of Influenza: recommendations of the Advisory Committee on Immunization Practices (ACIP). MMWR Recomm Rep 55 (RR-10): 1-42, 2006.

27. Weber DJ and Rutala WA: Immunization of immunocompromised persons. Immunol Allergy Clin North Am 23: 605-634, v-vi, 2003.

28. Fraser C, Donnelly CA, Cauchemez S, et al: Pandemic potential of a strain of influenza A (H1N1): early findings. Science 324: 1557-1561, 2009.

29. Dawood FS, Jain S, Finelli L, et al: Emergence of a novel swineorigin influenza A (H1N1) virus in humans. N Engl J Med 360: 2605-2615, 2009.

30. Sotelo $\mathrm{J}$ and Perez-Padilla R: The flu epidemic in Mexico: the challenges for doctors. BMJ 338: b1820, 2009.

31. Butler D: Swine flu goes global. Nature 458: 1082-1083, 2009.

32. Perez-Padilla R, de la Rosa-Zamboni D, Ponce de Leon S, et al: Pneumonia and respiratory failure from swine-origin influenza A (H1N1) in Mexico. N Engl J Med 361: 680-689, 2009.

33. World Health Organization: Influenza surveillance network. URL http://gamapserver.who.int/GlobalAtlas/DataQuery/ viewData.asp (Accessed June 29, 2010), 2010. 